\title{
Radiology websites: A few useful websites on chest radiology and imaging
}

\author{
IK Indrajit \\ Department of Radiodiagnosis \& Imaging, Command Hospital (Air Force), Bangalore-560 007, India \\ Correspondence: Dr. I K Indrajit, Department of Radiodiagnosis \& Imaging, Command Hospital (Air Force), Bangalore-560 007, India. \\ E-mail: inji63@gmail.com
}

1. George Simon's X-Ray Collection at http://myweb. lsbu.ac.uk/dirt/museum/g-topics.html is a site dealing with chest radiology. It is maintained by Ian Maddison, a radiologist, and has cases in cardiothoracic radiology, from the G. Simon collection, of originally produced for the Museum of the Royal College of Radiologists, in London. The site has many sections such as, 'Topics in the G. Simon collection of Cases in Cardiothoracic Radiology' and 'Pulmonary Radiology Teaching' sourced from http://myweb.lsbu.ac.uk/dirt/museum/ topics.html\#chest. There are absorbing sections on film viewing strategies, with particular attention paid to chest x-ray, septal lines, patterns of pulmonary edema, collapse, nodules, and lines. Finally, a review of the silhouette sign is also offered.

2. Basic Chest X-Ray Review at http://rad.usuhs.mil/ $\mathrm{rad} /$ chest_review/index.html is a website prepared by W. Carpenter, at the Uniformed Services University of the Health Sciences, Bethesda, Maryland. The format contains illustrated information on plain chest radiography under the following sections: soft tissues and bones, mediastinum, hila, and lungs and pleura.

3. Lobar Collapse Tutorial at http://www.radiology. co.uk/srs-x/tutors/collapse/tutorial.htm is an illustrative tutorial. Created by Dr A. Downie, it is designed to explain, the various types of lobar collapse seen on a plain chest film. Topics covered include silhouette sign, causes of collapse, general features of lobar collapse, normal PA and lateral films, anatomy of the lobes on normal films, and the various.

4. Thoracopedia is an imaging encyclopedia of pediatric thoracic disease authored by M.P. D'Alessandro from the Department of Radiology, University of Iowa College of Medicine. This educative portal presented at http://www.virtualpediatrichospital.org/providers/ TAP/Thoracopedia.shtml, has cases listed by knowns / unknowns, which are organized by disease type or by age or simply as unorganized unknowns.
5. Chest X-Ray.com at http://www.chestx-ray.com/ is a well-conceived website covering numerous areas within chest radiology. This site maintained by Jud W. Gurney, Department of Radiology, Nebraska Medical Center, has sections on research, education, practice, and research resources. Education focuses on lectures, unknown cases, and the journal club, while Practice Resources offers protocols, standards, reports, and guidelines. Sharpening its look and purpose, the site offers a handy list of evidence-based medicine tools, such as, Probability of Malignancy in SPN calculator at http:// www.chestx-ray.com/SPN/SPNProb.html. Research Resources is an interactive section, featuring Medline search, for specific searches of a dozen radiology and chest-related journals. Besides this, there are sections on critically evaluating literature using statistical tests, with an interactive facility for real-time evaluation of data.

6. Radiological and Pathological Correlation in Chest Disease is a student project from the Faculty of Medicine at McGill University. It is available at http://sprojects. mmi.mcgill.ca/radpath/molson3/default2.htm. It has sections based on pattern analysis, of entities such as solitary pulmonary nodule, multiple cavities, oligemia, masses, and pleural lesions. Similarly, a Web Tutorial for Chest Anatomy and Lung Malignancies is available at http://www.chestradiology.net/. There are two broad sections, covering chest anatomy and lung malignancies.

7. The Image of Sarcoidosis is an online tutorial authored by Terrence C. Demos, MD, from the Department of Radiology, Maywood, IL, and is available at http://www. meddean.luc.edu/Lumen/meded/Radio/sarc/sarc.htm. The material covers this multisystem granulomatous disease of unknown etiology at areas such as, diagnosis, pathology, clinical imaging, radiographic stages, thoracic lymphadenopathy, parenchymal lung disease, and extrathoracic disease.

8. ICU Chest Radiology at http://www.med-ed.virginia. edu/courses/rad/chest/ is a valuable website that covers the busy, but neglected area of ICU radiology. Created 
and maintained by Joss D. Fernandez, Spencer B. Gay, and Paul M. Dee at the University of Virginia Health Sciences Center, the website deals with $\mathrm{x}$-ray techniques, describes the normal ICU series, focuses on lines and tubes, describes air and fluid in the chest, and discusses lung processes.

9. An Introductory Primer of Pulmonary HRCT: Anatomic Approach with Pathological Correlation is a part of the Practical Pathology of Chest Disease created by Martha L. Warnock from the University of California, San Francisco. Available at http://pathhsw5m54.ucsf.edu/ ctpath/ctpathcontents.html, the website focuses on the technical aspects of HRCT, lung anatomy, basic HRCT patterns, linear abnormalities, nodules, consolidation, ground-glass opacity, and cysts / lucencies.

10. Interpretation of High-Resolution Chest CT at http:// www.med-ed.virginia.edu/Courses/rad/hrct/index. $\mathrm{htm}$ is authored by Spencer B. Gay and a team from the University of Virginia Health Sciences Center. This is a self-tutorial that employs a systematic approach in interpreting a high-resolution chest computed tomography $(\mathrm{CT})$. Illustrative descriptions of air trapping, bronchiectasis, centrilobular nodules, groundglass opacity, honeycombing, mosaic attenuation, nodules, septal thickening, tree-in-bud, and so on, are available here.

\section{Endpiece}

The Fleischner Society periodically prepares a glossary of terms for thoracic radiography and computed tomography. The third and updated glossary prepared by the Fleischner Society for Thoracic Imaging replaces the previously published versions of 1984 and 1996. Authored by Hansell et al., the latest version titled Fleischner Society: Glossary of Terms for Thoracic Imaging is available from the journal Radiology at http://radiology.rsna.org/content/246/3/697. full.

The Chest Learning Module from the website 'CTisUS' is available at http://www.ctisus.org/organsys/chest/index. $\mathrm{html}$. The module created by Fishman et al. from the Johns Hopkins University, features a variety of educative areas, including protocols, syllabus, teaching files, lectures, references, and gallery. Chest X-Ray by Arcot J. Chandrasekhar is available at http://www.lumen.luc.edu/ lumen/MedEd/medicine/pulmonar/cxr/cxr_f.htm.
Center for Interactive Digital Education in Radiology (CIDER) from Fundación Sante Fe de Bogotá, Colombia, has a collection of e-books, including Pulmonary Radiology: Basic Concepts, at http://www.cider.edu.co/2008/.

Thoracic Imaging on the Internet is an educative material available at http://www.auntminnie.com/ index.asp?sec=refandsub=thi, which is accessible after a mandatory registration. The website of two agencies that deal specifically with tuberculosis include the National Tuberculosis Institute at http://ntiindia.kar.nic.in/ and International Union against Tuberculosis and Lung Disease at http://www.theunion.org/.

In recent times, a new breed of online databases has emerged. These include the Technology Information Portal, which commenced with a dedicated Web portal on modalities such as magnetic resonance imaging (MRI) and Ultrasonography (USG). Arriving close on its heels comes now the Radiology-Technology Information Portal (Radiology-TIP.com) at http://www.radiology-tip. com/serv1.php?type=welcome. The site currently offers educative material on radiophysics, $\mathrm{CT}$, contrast, radiation and safety, and a host of other related topics in radiology.

A Web-based Tanner-Whitehouse test to estimate bone age, created by Lorentz Jäntschi and Sorana Bolboacă, is available at http://vl.academicdirect.ro/medical_informatics/ bone_age/v1.0/. The interactive system involves 'clickmatching' a scanned radiograph template of the radius, ulna, metacarpal, and proximal and middle phalanx bones. It then generates a stage of bone age and development.

Radguru at http://www.radguru.net/ is the teaching portal of Teleradiology Solutions, promoting learning and continuing updates for postgraduate (PG) students and practicing professionals in Radiology. Teleradiology Solutions has today emerged as a leader in providing Teleradiology support to hospitals across the globe. The teaching Web portal has a range of educative material covering the following areas: PG quizzes, case discussion and tutorials (in neuro-, chest, abdominal, and musculoskeletal radiology), case of the month, imaging signs, featured journal clubs, academic pearls, and pitfalls in reporting that are all based on day-to-day experiences and presentations at the Indian Radiological and Imaging Association (IRIA) meets.

Source of Support: Nil, Conflict of Interest: None declared. 